\title{
Reflexiones en torno al acceso al agua en el Perú, en el marco de la nueva Ley de Recursos Hídricos
}

\section{Thoughts on access to water in Peru within the new Water Resources Law framework}

\author{
LUCÍA RUIZ OSTOIC
}

\begin{abstract}
Resumen: El artículo toma como punto de partida la dificultad de abordar los temas relacionados con la gestión del agua en el Perú. Por ello, nos presenta un acercamiento al tema hídrico, explicándonos sus modalidades administrativas, los derechos que involucra y un análisis crítico de la Ley de Recursos Hídricos del año 2008. Finalmente, nos plantea la necesidad de analizar la gestión integrada del recurso hídrico comprendiendo integralmente la Ley General de Aguas y la actual Ley de Recursos Hídricos y fomentando el diálogo entre los diversos actores sociales involucrados para evitar futuros conflictos.
\end{abstract}

Palabras clave: recurso hídrico - gestión agua - Ley General de Aguas

Summary: The difficulty involved addressing issues related with water management in Peru is the article's starting point. Therefore, the water issue approach is introduced explaining its administrative procedures, the rights involved and making a critical analysis of 2008 Water Resources Law. Finally, the need for an integrated management analysis of the water resource is highlighted by integrally understanding the General Water Law as well as the current Water Resources Law, and encouraging dialogue among social actors involved in order to avoid future conflicts.

Key words: water resource - water management - General Water Law

CONTENIDO: I. INTRODUCCIÓN.- II. EL ACCESO AL AGUA PARA SATISFACCIÓN DE NECESIDADES PRIMARIAS O DE USO PRIMARIO NO REQUIERE CONTAR CON UN DERECHO ADMINISTRATIVO DE USO.- III. MODALIDADES ADMINISTRATIVAS DE ACCESO A LOS RECURSOS HÍDRICOS.- IV. LICENCIAS DE USO DE AGUA.- IV.1. LICENCIA EN BLOQUE.- IV.1.1. ANTECEDENTES.IV.1.2. SITUACIÓN ACTUAL DE LA LICENCIA EN BLOQUE.- IV.1.3. EL CASO DE LAS COMUNIDADES CAMPESINAS E INDÍGENAS.- IV.2. LICENCIA PROVISIONAL.- V. PERMISOS DE USO DE AGUA.- VI. AUTORIZACIONES DE USO DE AGUA.- VI.1. ALCANCES DE LAS AUTORIZACIONES DE USO DE AGUA.- VI.2. EMPLEO INADECUADO DEL TÉRMINO AUTORIZACIÓN DE USO DE AGUA.- VII. ¿SON LAS LICENCIAS, AUTORIZACIONES Y PERMISOS LOS ÚNICOS DERECHOS DE USO DE AGUA?.- VII.1. RESERVA DE USO DE AGUA:

* Abogada por la Pontificia Universidad Católica del Perú, con estudios de maestría en Administración de Negocios por CENTRUM PUCP y estudios de maestría en Conservación y Desarrollo Sostenible por la Universidad Peruana Cayetano Heredia. Correo electrónico: Iruizost @yahoo.com 
UN DERECHO ESPECIAL.- VIII. LICENCIA PROVISIONAL, AUTORIZACIÓN DE USO Y RESERVA: ¿SE PUEDEN USAR INDISTINTAMENTE?.- VIII.1. PLAZOS.VIII.2. USO EFECTIVO DEL RECURSO.- VIII.3. MOTIVOS PARA SU OTORGAMIENTO.- IX. REFLEXIONES FINALES.

\section{INTRODUCCIÓN}

No es fácil el tránsito hacia cambios estructurales cuando estos se relacionan con temas sensibles que involucran a porcentajes significativos de la población peruana. Este es el caso de los temas relacionados con la gestión del agua en el Perú, que son particularmente complejos por la diversidad de actores y sectores involucrados, así como por el impacto concreto que la gestión del recurso hídrico tiene en el ámbito rural. Un aspecto que merece atención y análisis es el del acceso al recurso hídrico y la solidez respecto de los derechos de agua otorgados, pues en cierta medida este conocimiento adecuado del tema puede prevenir o atenuar la conflictividad alrededor de la gestión del agua.

A inicios del siglo XX, la estructura económica latinoamericana giraba en torno a la agricultura y la extracción de recursos minerales, por lo que no es de extrañar que la primera norma en nuestro país regulando la gestión de las aguas, el Código de Aguas de 1902, planteara una relación tierraagua que hacía al propietario del primero, dueño del segundo. Es por esto que podemos encontrar, en los registros de propiedad o contratos de la primera mitad del siglo XX, referencias a transferencias de tierras que incluían además la transferencia del agua que nacía o discurría por ellas.

Esta situación se empieza a cuestionar hacia la segunda mitad del siglo XX y ya en el primer gobierno del arquitecto Fernando Belaunde, se gesta una nueva Ley de Aguas que se publicaría a inicios del gobierno del general Juan Velasco Alvarado, por lo que muchos la asocian con el cambio conceptual que acompañó dicha gestión, más aún si corresponde en el tiempo a la Ley de Reforma Agraria ${ }^{1}$. La Ley General de Aguas dada mediante decreto ley 17752 quebraba la relación tierra-agua, estableciendo en su artículo 1 que «Las aguas, sin excepción alguna, son de propiedad del Estado, y su dominio es inalienable e imprescriptible. No hay propiedad privada de las aguas ni derechos adquiridos sobre ellas. El uso justificado y racional del agua, solo puede ser otorgado en armonía con el interés social y el desarrollo del país»².

1 La Ley de Reforma Agraria fue emitida mediante decreto ley 17716 en junio de 1969, en tanto la Ley General de Aguas fue emitida mediante decreto ley 17752 en julio del mismo año. Si bien estas normas fueron promulgadas siendo presidente de facto el general Juan Velasco Alvarado, ambas se gestaron durante el gobierno del arquitecto Belaunde, con la participación de importantes juristas de la época.

2 Es importante entender este cambio normativo respecto del cual no se ha dado marcha atrás desde que fue establecido. Más adelante se hará mención al proceso de adecuación de derechos anteriores a la Ley General de Aguas, el cual, más de cuarenta años después, aún no ha concluido. 
Si bien esta norma genera un quiebre conceptual en un contexto en que la propiedad de amplias extensiones agrícolas era también cuestionada, no significa que se alejó de la visión agrarista del anterior Código; por el contrario, ha sido por muchos catalogada como una norma de visión claramente sectorial, además de centralista. Basta una rápida mirada a la norma para apreciar que la misma tiene un enfoque básicamente agrario, con pocas referencias a usos de agua además de la citada, así como con una fuerte presencia estatal en todo lo relacionado con el desarrollo de infraestructura y toma de decisiones.

Con el fin de implementar lo establecido en el artículo 1 de la Ley General de Aguas, la norma establece modalidades administrativas de acceso al recurso hídrico, denominadas licencias, autorizaciones y permisos, ordenando la adecuación universal de los usuarios de agua a este nuevo sistema ${ }^{3}$ y dejando sin efecto, por lo tanto, toda referencia a derechos de agua contenidos en documentos de propiedad de tierras. A pesar de que en la década de 1990 se emite una nueva constitución que plantea el acceso a los recursos naturales mediante la modalidad de concesión, la ley orgánica para el aprovechamiento de los recursos naturales de 1997 precisa la vigencia de las modalidades de acceso en materia de aguas a que se refería la Ley General de Aguas ${ }^{4}$.

Toda persona, sea esta natural o jurídica, privada o pública, para hacer uso del recurso agua, requiere contar con un derecho de uso previamente otorgado por la autoridad correspondiente ${ }^{5}$. Es necesario reiterar esta afirmación, vigente hace más de cuarenta años, aun en nuestros días, pues la realidad es que la gran mayoría de usuarios ha hecho uso del recurso hídrico durante décadas sin contar con un derecho administrativo que lo respalde, incluyendo algunas instituciones del Estado ${ }^{6}$.

Si bien la emisión de la Ley General de Aguas de 1969 generó un cambio fundamental respecto a la relación «Estado-agua-usuario del recurso», ordenando la adecuación universal a las nuevas modalidades de acceso, no se asigna un plazo perentorio para ello y menos una sanción a quien incumpla en adecuarse. Esta situación implicó que durante cuatro décadas se ejerciera un uso informal del agua en el Perú, con solo un cinco por ciento de los derechos de uso de agua regularizados, debido, en un inicio, al sistema centralizado de trámite y otorgamiento de licencias ${ }^{7}$

3 Artículo 144 (disposición transitoria) de la Ley General de Aguas, decreto ley 17752.

4 Artículos 19 y 24 de la ley orgánica para el aprovechamiento de los recursos naturales, ley 26821.

5 Más adelante explicaremos que existe una excepción a esta afirmación, pues la satisfacción de necesidades primarias o de uso primario no requiere contar con derechos administrativos de uso.

6 Algunas importantes obras de infraestructura hidráulica poblacionales han carecido de derechos de uso de agua, tanto en el proceso de su construcción como al finalizar este. Un claro caso de ello es el de las Empresas Prestadoras de Servicios de Saneamiento, muchas de las cuales han tramitado y obtenido los derechos de uso de agua respecto de las aguas que gestionaban, durante la segunda mitad de la década de 1990.

7 Las licencias y autorizaciones de agua, de las que trataremos más adelante, eran otorgadas entre 1969 y 1981 mediante resolución suprema y resolución ministerial, respectivamente.

REFLEXIONES

EN TORNO AL

ACCESO AL AGUA

EN EL PERÚ, EN

EL MARCO DE

LA NUEVA LEY

DE RECURSOS

HÍDRICOS

THOUGHTS ON

ACCESS TO WATER

IN PERU WITHIN

THE NEW WATER

RESOURCES LAW

FRAMEWORK 
y, posteriormente, a un fuerte manejo gremial por parte de las Juntas de Usuarios de Distritos de Riego ${ }^{8}$.

Es recién en el año 2004 que el gobierno de la época inicia un agresivo proceso de formalización de derechos, mediante el Programa de Formalización de Derechos de Uso de Agua con Fines Agrícolas (PROFODUA $)^{9}$, generándose para ello un mecanismo que luego sería recogido por la actual Ley de Recursos Hídricos: la asignación de agua en bloque, que devendría en lo que hoy se conoce como las licencias de uso de agua en bloque. Este proceso de formalización de derechos pretendía regularizar masivamente los derechos de uso de agua agrario considerando que el ochenta por ciento del uso consuntivo del agua ${ }^{10} \mathrm{en}$ el Perú es con estos fines, lo cual permitiría, tiempo después, contar con un Registro Administrativo de Derechos de Uso de Agua ${ }^{11}$ en el que se inscribieran de oficio los derechos que se otorgan.

En el 2008 se promulga la Ley de Recursos Hídricos (ley 29338) después de más de una década de desarrollar plataformas de discusión a nivel nacional, grupos de trabajo especializados y contar con diversas propuestas normativas desde los Poderes Ejecutivo y Legislativo. Esta norma mantiene las denominaciones de las modalidades de acceso al recurso hídrico, pero les confiere características diferentes a las planteadas por la Ley General de Aguas; además de reconocer el hecho de que la informalidad en el uso del recurso es latente, por lo que plantea

8 Después de un periodo de exclusiva decisión por parte del Estado respecto del otorgamiento de los derechos de uso, c0on la dación del decreto legislativo 653 de 1991 se otorga a las Juntas de Usuarios de Distrito de Riego (integradas legalmente por usuarios agrarios y no agrarios, pero en la realidad dirigidas solo por los primeros) la facultad de opinar previamente y con carácter vinculante, respecto del otorgamiento de usos de agua en general, generando con ello un poder de decisión que usado incorrectamente generó trabas al otorgamiento de usos no agrarios, e incluso élites y corrupción en el caso del otorgamiento de usos agrarios.

9 Mediante decreto supremo 041-2004-AG, promulgado en noviembre de 2004, se dictan disposiciones para la ejecución del Programa Extraordinario de Formalización de Derechos de Uso de Agua con Fines Agrarios (PROFODUA) cuya finalidad era otorgar de forma masiva derechos de uso de agua agrario, inicialmente en valles costeros, con financiamiento de la cooperación internacional. Considerando que los derechos de uso de agua estaban contenidos en los títulos de propiedad antes de 1969 y que producto de la reforma agraria se expropiaron y fraccionaron propiedades, las cuales después de décadas habían sido transferidas a descendientes o nuevos propietarios, además que en materia de aguas se estableció que los derechos se otorgarían por el Estado, hacia el año 2004 era mínimo el número de usuarios agrarios con un derecho de uso de agua otorgado por la autoridad competente, efectuándose el uso del recurso sobre la base de lo establecido en documentos internos de las Juntas de Usuarios de Distrito de Riego denominado «Padrón de Usuarios».

$10 \mathrm{El}$ uso consuntivo del agua es aquel en el que el recurso se consume en el proceso para el cual se utiliza; y aun cuando se pueda recuperar parte del recurso, no se obtendrá en el volumen o calidad original. En el caso del uso agrario, cuando se irrigan las tierras, una parte del recurso es absorbido por las plantas, una parte se evapora en el traslado producto de las condiciones climáticas, una parte se filtra por el suelo (en mayor o menor medida dependiendo de sus características físicas) hacia los acuíferos subterráneos y una parte drena de manera superficial hacia las zonas bajas del valle. Incluso en caso se recupere parte del recurso aguas abajo, se debe evaluar si las características físicas son las mismas, pues dependiendo del uso o no de químicos en los procesos agrícolas, el agua transportará estos elementos hacia su nuevo destino, contando ahora con un recurso que varía sus características físico-químicas. Este ejercicio puede aplicarse a otros usos consuntivos, como el minero, el industrial, el poblacional y demás.

11 Mediante decreto supremo 021-2007-AG se crea el Registro Administrativo de Derechos de Uso de Agua (RADA) con la finalidad de inscribir en forma diferenciada a nivel nacional las licencias, autorizaciones y permisos para el uso de agua, con sus respectivas actualizaciones y extinciones. 
facilitar la regularización de los derechos administrativos de uso de agua a quienes muestren su uso público, pacífico y continuo por cinco años o más ${ }^{12}$.

\section{EL ACCESO AL AGUA PARA SATISFACCIÓN DE NECESIDADES PRIMARIAS O DE USO PRIMARIO NO REQUIERE CONTAR CON UN DERECHO ADMINISTRATIVO DE USO}

Tanto en la norma de 1969 como en la de 2008 se hace referencia a que la satisfacción de necesidades primarias no requiere contar con un derecho administrativo para el uso del recurso hídrico, por lo que es fundamental entender a qué nos referimos con este término.

Lo que en la Ley de Aguas se denominaba necesidades primarias ${ }^{13}$, la Ley de Recursos Hídricos lo denomina uso primario ${ }^{14}$ y lo define claramente como la utilización directa y efectiva del recurso hídrico, en las fuentes naturales y cauces públicos de agua, con el fin de satisfacer necesidades humanas primarias de preparación de alimentos, consumo directo y aseo personal, incluyendo en el concepto de uso primario aquellos de índole cultural, religiosa y ritual. La incorporación de este importante componente cultural ha sido un aporte normativo fundamental en un país multicultural como el nuestro, en el que la poca flexibilidad de algunas autoridades y su desconocimiento de las tradiciones y cosmovisión local, generó una innecesaria escalada de conflictos en ocasiones anteriores.

Adicionalmente, para considerarse este uso como primario, no debe tener fin lucrativo, alterar las fuentes de agua de las que se obtiene ni ser ejercido mediante medios diferentes a los estrictamente manuales. Este es un aspecto clave para que diferenciemos, como lo hicieron las normas de aguas de 1969 y 2008, entre el uso primario o necesidades primarias y el denominado uso poblacional del recurso. El uso poblacional del recurso hídrico requiere contar con un derecho administrativo de uso de agua para ser ejercido y está relacionado con el abastecimiento de agua a través de una infraestructura de redes, para lo que se requiere que una organización o institución brinde dicho servicio, previo tratamiento del agua que permita su consumo seguro. Como es evidente, el uso poblacional del recurso hídrico, sea a nivel rural ${ }^{15} \mathrm{o}$ urbano ${ }^{16}$, no se

12 Segunda disposición complementaria y final de la Ley de Recursos Hídricos, ley 29338.

13 Artículo 8 de la Ley General de Aguas, decreto ley 17752.

14 Artículos 36 y 37 de la Ley de Recursos Hídricos, ley 29338.

$15 \mathrm{En}$ el ámbito rural encontramos a las Juntas Administradoras de Servicios de Saneamiento, denominadas JASS, como las organizaciones autorizadas por la Ley de Servicios de Saneamiento (ley 26338) para brindar a las poblaciones menores los servicios de captación y distribución de agua. 16 En el ámbito urbano encontramos a las Empresas de Saneamiento, denominadas EPS, como las instituciones autorizadas por la Ley de Servicios de Saneamiento (ley 26338) para brindar los servicios de captación y distribución de agua, así como recolección y disposición final de aguas residuales domesticas.

REFLEXIONES

EN TORNO AL

ACCESO AL AGUA

EN EL PERÚ, EN

EL MARCO DE

LA NUEVA LEY

DE RECURSOS

HÍDRICOS

THOUGHTS ON

ACCESS TO WATER

IN PERU WITHIN

THE NEW WATER

RESOURCES LAW

FRAMEWORK 
efectúa mediante métodos manuales para la captación del recurso ni para su distribución, además que tratándose de un servicio brindado, implica una contraprestación económica por parte de los usuarios finales del servicio.

Finalmente, al Estado le corresponde garantizar este acceso libre a las fuentes naturales y cauces artificiales públicos, estableciendo, de ser necesario, lugares o zonas de libre acceso ${ }^{17}$.

\section{MODALIDADES ADMINISTRATIVAS DE ACCESO A LOS RECURSOS HÍDRICOS}

Las modalidades administrativas de acceso al recurso hídrico en nuestro país son denominadas de manera genérica derechos de uso de agua ${ }^{18} \mathrm{y}$ fueron instituidas por la Ley General de Aguas de 1969, reconocida su vigencia por la Ley Orgánica de Aprovechamiento Sostenible de los Recursos Naturales de 1997 y recogidas por la actual Ley de Recursos Hídricos de 2008.

Los derechos de uso de agua son de tres clases y si bien las denominaciones utilizadas desde el año 1969 son las mismas y las condiciones generales que los caracterizan también, no así las características específicas de cada uno de estos derechos de uso que han variado desde su formulación en la ley que les dio origen y la actual ley que los recoge. Antes de analizar cada una de las clases de derechos de uso, mencionaremos las condiciones generales de los mismos.

A. Los derechos de uso de agua se otorgan y ejercen considerando la disponibilidad del recurso, tanto física como administrativa

Se entiende que el recurso hídrico es producto de un ciclo hidrológico vulnerable a condiciones naturales y humanas, por lo que los derechos sobre el mismo se otorgan y ejercen considerando las condiciones de disponibilidad respecto a la cantidad, calidad y oportunidad ${ }^{19}$ del recurso. Es por este motivo que los derechos de uso de agua se otorgan expresados en volúmenes anuales de disposición mensual y requieren de parte del usuario, en la mayoría de casos, la generación de infraestructura hidráulica de regulación, es decir, contar con reservorios que permitan hacer un uso efectivo del recurso de acuerdo con las necesidades de la actividad que realiza el usuario del recurso y no con lo que el ciclo hidrológico determine. Además, ante situaciones de escasez

17 Artículo 39 de la Ley General de Aguas, decreto ley 17752.

18 Título IV de la Ley de Recursos Hídricos, ley 29338.

19 De los criterios de cantidad, calidad y oportunidad que deben tenerse en cuenta al solicitar u otorgar un derecho de uso de agua, el de oportunidad se refiere a la temporalidad en la presencia del recurso. En el caso del agua superficial (aquella que discurre a nivel continental), existe una dependencia respecto del periodo de precipitaciones en la parte alta y media de la cuenca, en tanto respecto del agua subterránea (acuíferos) o de mar, se debe considerar la cantidad y calidad del recurso además de la capacidad de recarga en el primer caso y aspectos ecosistémicos en el segundo. 
del recurso, se establecen prioridades en el acceso al mismo, teniendo siempre preferencia el uso primario y el uso poblacional, dando paso luego a los usos productivos, entre los cuales los usos agrario, acuícola y pesquero tienen prioridad en tanto están relacionados con la seguridad alimentaria.

Quien esté interesado en acceder a un volumen de agua para ser utilizado en la actividad que determine, debe probar la disponibilidad física del recurso, pero además su disponibilidad administrativa. Es decir, sustentar la existencia del recurso y demostrar que no existen derechos preexistentes respecto del volumen de agua que desea utilizar. Sustentar la disponibilidad administrativa del recurso debería ser innecesario, si como país contáramos con un adecuado registro de derechos de uso de agua que consigne la totalidad de derechos otorgados. Sin embargo, debemos entender que no contamos con una información oficial respecto de la oferta hídrica nacional y contamos aún con un elevado número de usuarios de agua que no cuenta con un derecho administrativo que lo respalde, situación que no solo se da respecto del uso agrícola.

\section{B. Los derechos de uso de agua se otorgan para un uso o actividad en particular} y deben ser ejercidos en un lugar determinado

Un derecho de agua se otorga para un uso $^{20}$ determinado, que puede ser poblacional o productivo, siempre que se cumpla con la normatividad vigente. Sin embargo, una vez otorgado un derecho para un uso especifico, no puede ser ejercido en una actividad diferente a aquella para la cual se otorgó. La misma situación ocurre respecto al lugar en que el derecho será ejercido, pues el uso para el que fue otorgado y el lugar en que se debe ejercer son indesligables, acarreando el incumplimiento de estas disposiciones la resolución de la licencia, permiso o autorización.

Es importante tener claro, entonces, que el derecho otorgado seguirá vigente en tanto subsista el uso para el cual fue otorgado y este se ejerza en el lugar para el que fue solicitado, no así si alguno de estos dos componentes es variado. Todo cambio implica un nuevo otorgamiento de derecho por parte de la autoridad administrativa.

\section{Los derechos de uso de agua no son transferibles}

En el Perú este es un aspecto clave en materia de agua, pues a diferencia de la regulación respecto a otros recursos naturales, en el caso del recurso hídrico se ha establecido claramente la imposibilidad de transferir los derechos que se tengan sobre él. En caso el titular del derecho decida

REFLEXIONES

EN TORNO AL

ACCESO AL AGUA

EN EL PERÚ, EN

el MARCO DE

LA NUEVA LEY

DE RECURSOS

HÍDRICOS

THOUGHTS ON

ACCESS TO WATER

IN PERU WITHIN

THE NEW WATER

RESOURCES LAW

FRAMEWORK 
no seguir haciendo uso de él, debe revertirlo al Estado, no pudiendo mediante un acuerdo entre privados hacer una transferencia del mismo como sí ocurre respecto de los derechos forestales o mineros, para poner ejemplos de recursos renovables y no renovables.

Sin embargo, la normatividad vigente permite que el nuevo titular de un predio, establecimiento o actividad tenga un derecho preferente de acceder al recurso hídrico ${ }^{21}$, lo cual en materia agraria como en cualquier otra actividad económica es fundamental, ya que no podría pensarse en la transferencia, por ejemplo, de terrenos de cultivo sin considerar que estos requieren contar con el recurso hídrico asociado. De no efectuarse de esta manera, cualquier transferencia de derechos entre privados acarrea la resolución administrativa del derecho otorgado.

D. Los derechos de uso de agua se otorgan, suspenden, modifican o extinguen por resolución administrativa, correspondiendo al Estado garantizar el cumplimiento de los derechos otorgados

Las clases de derechos de uso de agua son tres desde hace más de cincuenta años en el Perú: licencias, permisos y autorizaciones de uso de agua. Si bien se han conservado las denominaciones utilizadas, las características especificas de cada clase de derecho han variado e incluso se han establecido subclases, que en algunos casos vulneran la razón de ser del derecho de uso al cual corresponden.

A efectos jurídicos, esto podría no tener mayores implicancias en tanto la legislación actual es clara respecto de las características y vigencia de los nuevos conceptos que definen a los derechos de uso de agua. Sin embargo, podría tenerlas a efectos prácticos, pues la diversidad de sectores interactuando requieren un conocimiento integral e histórico que permita una similar comprensión de los conceptos empleados ${ }^{22}$.

\section{LICENCIAS DE USO DE AGUA}

La licencia es el derecho administrativo por excelencia para acceder al recurso hídrico, aquel al que aspiran quienes desean un derecho de uso de agua para realizar una actividad de carácter permanente, pues permite usar el recurso por plazo indeterminado, subsistiendo la licencia en tanto subsista la actividad para la que fue otorgada, en el lugar en que se determinó ejercer.

21 Artículo 65, numeral 3 del reglamento de la Ley de Recursos Hídricos, decreto supremo 001-2010-AG. 22 En tanto el ochenta por ciento del recurso hídrico utilizado a nivel nacional es con fines agrarios y los usuarios de los mismos se cuentan por cientos de miles a diferencia de quienes hacen uso del recurso para otros fines, es necesario tener claro que los interlocutores en todos los casos comprenden conceptualmente a qué se refiere cada quien al mencionar licencias, permisos o autorizaciones, considerando sobre todo que el concepto original ha sido utilizado por cuarenta años a diferencia del nuevo que solo tiene cinco de existencia y puede no haber sido adecuadamente difundido. 
Las licencias pueden otorgarse para usos consuntivos o no consuntivos del recurso hídrico ${ }^{23}$, así como para el uso de aguas superficiales o subterráneas. Mediante una licencia se faculta a usar y registrar una dotación anual de agua en metros cúbicos, a ejercer acciones legales para su defensa, además de dar la potestad de efectuar inversiones en el tratamiento, transformación y reutilización del recurso para el uso al que fue otorgado. Este derecho es otorgado actualmente por la autoridad administrativa del agua.

Esta modalidad de acceso tiene en esencia las mismas características desde que fue creada por la Ley General de Aguas en 1969. Sin embargo, la Ley de Recursos Hídricos añade dos submodalidades, una producto de los procesos de formalización masiva de derechos de uso (licencia en bloque) y otra que denota la intención de apoyar los procesos de inversión en concesiones de diverso tipo (licencia provisional), pero que a su vez se distancia del concepto original de licencia.

\section{IV.1. Licencia en bloque}

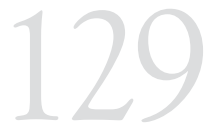

REFLEXIONES EN TORNO AL ACCESO AL AGUA EN EL PERÚ, EN EL MARCO DE LA NUEVA LEY DE RECURSOS HÍDRICOS

THOUGHTS ON ACCESS TO WATER IN PERU WITHIN THE NEW WATER RESOURCES LAW FRAMEWORK

\section{IV.1.1. Antecedentes}

Los antecedentes de la licencia de uso de agua en bloque se remontan al año 2004, en el marco de un necesario proceso impulsado por el gobierno peruano para realizar la formalización masiva de los derechos de uso de agua con fines agrarios en el ámbito rural, situación que había sido relegada por décadas. Ante la necesidad de formalizar los derechos de uso de agua con fines agrarios y la complejidad de otorgar cientos de miles de licencias y permisos de uso de agua, se diseña un programa masivo y gratuito de formalización de derechos de uso de agua denominado PROFODUA ${ }^{24}$, en virtud del cual la autoridad de aguas de la época ${ }^{25}$ decidió agrupar a los usuarios a ser formalizados, según la fuente de agua de la que se abastecían y la infraestructura hidráulica menor de riego que compartían, otorgándoles una asignación de agua en bloque ${ }^{26}$.

Esta primera experiencia de formalización masiva, que contó con una importante plataforma informática para efectos de la georreferenciación de los predios agrícolas y el registro de los derechos otorgados, fue satisfactoria, aun cuando las resoluciones que otorgaban las licencias o permisos contaban con frondosos anexos que detallaban listados de más de cien usuarios y consignaban en cada caso los volúmenes de agua asignados individualmente y la ubicación de los predios en los que

23 El uso no consuntivo del agua es aquel en el que el recurso no se consume en el proceso para el cual se utiliza, debiendo por lo tanto devolverse el recurso en la cantidad y calidad en que fue captado. El uso no consuntivo de agua por excelencia es el energético, pues se emplea la fuerza y volumen del agua para producir energía, sin que ello implique una modificación de volumen o calidad del recurso desde que es captado hasta que es devuelto nuevamente al cauce de los ríos.

24 Programa Extraordinario de Formalización de Derechos de Uso de Agua con Fines Agrarios.

25 Intendencia de Recursos Hídricos del Instituto Nacional de Recursos Naturales.

26 Artículo 7 del decreto supremo 041-2004-AG, que dicta disposiciones para la ejecución del PROFODUA. 
estos derechos debían ser ejercidos. Un aspecto, sin embargo, requería ser evaluado: se estaba otorgando una licencia a un grupo de personas individualizando volúmenes y predios agrarios, pero la distribución del recurso no tenía un responsable claro, aun cuando la mayoría de infraestructuras menores de riego se administraran a través de comités o comisiones de regantes, organizaciones de usuarios de agua a cargo de su operación y mantenimiento ${ }^{27}$.

En el caso de las comunidades campesinas, por el contrario, se otorgaba la licencia en bloque a nombre de la comunidad, detallando en los anexos la lista de comuneros beneficiados, los volúmenes de agua asignados en cada caso y la ubicación de los predios. En este caso se tenía claro a un responsable que coordinaba al grupo de usuarios y se hacía cargo de la distribución del recurso, así como del mantenimiento de la infraestructura.

Estas experiencias previas permitieron que, al elaborarse la Ley de Recursos Hídricos, la licencia en bloque ya contara con una serie de lecciones aprendidas que permitieron mejorar ciertos aspectos de esta figura jurídica.

\section{IV.1.2. Situación actual de la licencia en bloque}

Según lo establece la actual Ley de Recursos Hídricos, la licencia en bloque se otorga a una organización de usuarios de agua reconocida ${ }^{28}$, a la cual le corresponde la operación y mantenimiento de la infraestructura hidráulica, así como la distribución del recurso entre quienes son señalados por la licencia en bloque como beneficiarios de la misma. Los beneficiarios de la licencia en bloque pertenecen a la organización a cuyo nombre se expide la licencia y comparten una fuente e infraestructura hidráulica común.

Posteriormente al otorgamiento de la licencia en bloque, corresponde a la organización de usuarios que la reciba emitir Certificados Nominativos que reflejen el derecho de uso de agua de sus miembros, representando estos documentos la parte de la asignación de agua que corresponde a cada uno de los integrantes del bloque y otorgando a sus titulares, respecto de la parte que les corresponde, los mismos derechos y obligaciones que una licencia individual de uso de agua.

27 Las organizaciones de usuarios de agua de uso agrario, a lo largo de los últimos cincuenta años, han estado a cargo de la gestión de la infraestructura menor de riego, existiendo tres niveles de organización: juntas, comisiones y comités de usuarios de agua de uso agrario. Las juntas de usuarios se organizan sobre la base de un sistema hidráulico común y se encargan de la operación y mantenimiento de la infraestructura hidráulica, de la distribución del agua, además del cobro y administración de las tarifas de agua. Estas juntas de usuarios están conformadas a su vez por comisiones. Por su parte, los comités de usuarios, que pueden ser de aguas superficiales, de aguas subterráneas y de aguas de filtración, se organizan a nivel de canales menores, a nivel de pozo, y a nivel de área de afloramiento superficial, respectivamente.

28 Las organizaciones de usuarios de agua son reconocidas administrativamente por la autoridad de agua. 
En tanto se trata de la instancia responsable de la gestión del recurso, por haber sido otorgado este a su nombre en bloque, esta situación permite que la organización de usuarios supervise las transferencias de terrenos agrícolas, sea por motivos sucesorios o comerciales, manteniendo informada a la autoridad y permitiendo al adquirente ejercer el derecho preferente en la asignación de nuevas licencias.

\section{IV.1.3. El caso de las comunidades campesinas e indígenas}

Como se menciona en el acápite referido a los antecedentes de la licencia en bloque, las comunidades campesinas y nativas constituyeron un ejemplo a seguir respecto a la asignación de derechos en bloque, en tanto estas organizaciones recibían los derechos de uso de agua y se encargaban de gestionar el recurso a favor de sus miembros, antes que la norma vigente lo estableciera de la misma manera respecto de las organizaciones de usuarios de agua y sus miembros. La actual Ley de Recursos Hídricos reconoce que las comunidades campesinas y nativas se organizan en torno a sus fuentes naturales, microcuencas y subcuencas de acuerdo con sus usos y costumbres, teniendo a efectos del tema hídrico los mismos derechos de las organizaciones de usuarios de agua, sin necesidad de tener que constituir una organización diferente o superpuesta a la que tradicionalmente poseen.

Puede, sin embargo, inducir a error que la legislación de aguas vigente, al referirse a otros derechos de uso de agua ${ }^{29}$, mencione a las comunidades campesinas y nativas y a que el Estado reconoce y respeta el derecho de las mismas:

[...] a utilizar las aguas existentes o que discurren por sus tierras, así como sobre las cuencas de donde nacen dichas aguas, tanto para fines económicos, de transporte, de supervivencia y culturales, en el marco de lo establecido en la Constitución Política del Perú, la normativa sobre comunidades y la ley ${ }^{30}$.

Una lectura ligera podría dar a entender que este reconocimiento de derechos genera una nueva modalidad administrativa de acceso al recurso hídrico, distinta a las mencionadas o, en una interpretación aun más compleja, que las comunidades campesinas y nativas no requieren tramitar la asignación de derechos de uso de agua, por su calidad de comunidades.

$\mathrm{Al}$ respecto es importante recordar que el ejercicio de los derechos de las comunidades sobre las aguas se ha enmarcado expresamente en

29 Capítulo III del título IV de la Ley de Recursos Hídricos, ley 29338, denominado «otros usos de agua".

30 Artículo 64 de la Ley de Recursos Hídricos, ley 29338. 
la Constitución peruana y las normas especiales sobre comunidades y recurso hídrico. Si bien el derecho que tienen las comunidades de acceder al recurso hídricos es imprescriptible, prevalece respecto de terceros, se ejerce de acuerdo con los usos y costumbres ancestrales de cada comunidad y de ninguna manera menoscabará los derechos reconocidos por el Convenio 169 de la Organización Internacional del Trabajo; no por ello carecerá de una formalidad administrativa otorgada por el Estado en aquellos casos que lo requieran. Como se ha mencionado, para hacer uso del recurso hídrico, toda persona natural o jurídica, pública o privada requiere obtener un derecho de uso, con excepción del uso primario mediante el cual se satisfacen necesidades primarias, además de las de índole cultural. Esto implicaría, en el caso de las comunidades, que requieren obtener derechos de uso de agua para aquellas actividades que no son de uso primario, bajo una modalidad de licencia en bloque.

\section{IV.2. Licencia provisional}

$\mathrm{Al}$ evaluar la figura de la licencia provisional, como está planteada en la Ley de Recursos Hídricos, se aprecia de inmediato que esta se aleja de las características de la licencia de uso de aguas y se confunde con otras modalidades de acceso administrativo al recurso, diferentes de las licencias. El principal criterio para hacer una afirmación de este tipo es que la licencia provisional quiebra la esencia fundamental de la licencia de uso de agua: su plazo indeterminado. La licencia de uso en materia de aguas tiene por característica principal que se otorga por plazo indeterminado y para realizar una actividad permanente, por lo que no debería existir una «licencia provisional».

Durante la década de 1990, la promoción de inversiones privadas en el Perú impulsada desde el gobierno central se encontró con una limitante en materia de acceso a los recursos hídricos, pues la Ley General de Aguas consideraba al Estado como el principal propulsor de infraestructura hidráulica de todo tipo, mencionando la participación de los privados como marginal. En caso el Estado hubiera querido construir infraestructura vinculada con la regulación del recurso hídrico y su posterior uso $^{31}$, la Ley General de Aguas consideraba la modalidad de «reserva de agua» ${ }^{32}$ con fines de interés público, que permitía, como su nombre lo indica, reservar el recurso hídrico para ser utilizado una vez concluida la obra. En otras palabras, se establecía que un determinado volumen de agua disponible físicamente no sería otorgado a terceros mediante ningún procedimiento administrativo en tanto no concluyera 
el Estado la obra para el cual lo había reservado, que por ser de iniciativa estatal se presumía era de interés público.

El inversionista privado, por su parte, no podía hacer uso de la figura de la reserva por la presunción que lo guiaba el interés particular, lo cual motivó un conflicto normativo entre la Ley General de Aguas y la frondosa normatividad minera, energética y agroindustrial que se desarrolló con miras a promover la inversión privada en el país. En caso un inversionista privado quisiera desarrollar una obra de infraestructura que, entre estudios y construcción, pudiera tomar de cuatro a siete años, le era prácticamente imposible obtener un derecho de uso de agua.

En el marco de la Ley General de Aguas, el uso justificado y racional del agua debía ser otorgado en armonía con el interés social y el desarrollo del país, por lo que los procedimientos establecidos para su otorgamiento no dejaban alternativa a lo que podía entenderse como «especulación» respecto del recurso. Quien solicitara una licencia de uso de agua no solo debía mostrar la disponibilidad física y administrativa del recurso, sino además hacer uso efectivo del mismo, puesto que era causal de caducidad $^{33}$ no solo no pagar periódicamente las denominadas tarifas de agua por el volumen otorgado, sino también el no hacer uso efectivo del recurso.

La situación se volvió más compleja a fines de la década de 1990 en el caso de las concesiones eléctricas, pues la autoridad de electricidad solicitaba que el derecho de agua estuviera asegurado por el inversionista, con anterioridad al otorgamiento de la concesión eléctrica y por ende de la construcción de la infraestructura con fines hidroeléctricos. Ante esta situación, la autoridad de aguas planteó el otorgamiento de licencias de agua condicionadas al otorgamiento de la concesión eléctrica definitiva, así como de la conclusión de la obra de infraestructura a que se refería la concesión otorgada o por otorgarse.

Como puede apreciarse, ya en la referida década se buscaban alternativas ante la incompatibilidad entre la normativa de promoción de inversiones y la normativa sectorial en materia de agua, pero enmarcadas en las características propias de la licencia: se otorgaba de manera condicionada al cumplimiento de determinados aspectos y en caso no se cumplieran, esta se declaraba concluida. En la actualidad, la normatividad establece que las licencias de uso de agua se otorgan con posterioridad a la autorización que otorgue la autoridad sectorial competente para realizar la actividad en la que se utilizará el recurso ${ }^{34}$.

REFLEXIONES

EN TORNO AL

ACCESO AL AGUA

EN EL PERÚ, EN

EL MARCO DE

LA NUEVA LEY

DE RECURSOS

HÍDRICOS

THOUGHTS ON

ACCESS TO WATER

IN PERU WITHIN

THE NEW WATER

RESOURCES LAW

FRAMEWORK 
Sin embargo, en el marco de la Ley de Recursos Hídricos, la denominada licencia provisional se otorga a titulares de concesiones obtenidas ante las entidades públicas competentes, que tengan como fin la realización de estudios en cualquier actividad, su plazo es determinado y no puede superar el de las concesiones que la originan. Una vez cumplidas las condiciones bajo las cuales se otorgó la licencia provisional se procede, a solicitud de parte, al otorgamiento de la licencia que faculte a su titular para hacer uso efectivo del agua. Si esta disposición está clara, ipara que una licencia provisional?

Más adelante veremos que esta figura guarda grandes similitudes con el derecho de uso de agua denominado autorización de uso y con el derecho especial denominado reserva de agua.

\section{PERMISOS DE USO DE AGUA}

Esta modalidad de acceso al recurso hídrico es la que ha sufrido un cambio significativo entre su creación por la Ley General de Aguas y su actual definición por parte de la Ley de Recursos Hídricos.

La Ley General de Aguas de 1969 señalaba que los permisos de uso de agua se otorgaban respecto de recursos sobrantes, supeditados a la eventual disponibilidad de las aguas y en el caso del uso agrícola, condicionados a determinados cultivos ${ }^{35}$. También establecía que este derecho de agua era de carácter temporal, debiendo ser solicitado y otorgado anualmente en virtud a la situación hídrica proyectada; no generándose responsabilidad por parte de la autoridad de aguas en caso se dieran pérdidas generadas por la ausencia del recurso.

La actual Ley de Recursos Hídricos establece que los permisos ${ }^{36}$ se clasifican en dos clases: (a) el permiso de uso de agua para épocas de superávit hídrico que otorga al titular la facultad de usar una cantidad de agua proveniente de una fuente natural, cuando la autoridad de aguas haya declarado el estado de superávit hídrico una vez cumplidos los compromisos con quienes tienen licencia de uso de agua en el sector o distrito hidráulico; (b) el permiso de uso sobre aguas residuales que otorga a su titular la facultad de usar una cantidad de agua proveniente de aguas superficiales de retorno, filtraciones o drenajes, resultantes del ejercicio del derecho de los titulares de licencias de uso.

Son derechos de duración indeterminada pero de ejercicio eventual. Este constituye el aporte principal por parte de la nueva ley, en tanto el usuario puede obtener un derecho de uso por una sola vez y hacerlo efectivo en la medida de la disponibilidad del recurso durante todo 
el tiempo que decida ejercer su derecho, reduciendo los costos de transacción de manera significativa ${ }^{37}$.

Estos permisos se otorgan mayoritariamente para uso agrario. La redacción, tanto de la Ley General de Aguas como de la Ley de Recursos Hídricos, permite interpretar que estos permisos pueden ser otorgados para usos diferentes al agrario, sin embargo, los usuarios de este tipo de derecho son básicamente agricultores. En cualquier otra actividad económica, sería complejo depender de agua que no se tiene seguridad si estará disponible, en tanto en la actividad agrícola se cuenta con la opción del cultivo temporal, que permite comprometer el riego para periodos cortos de siembra y cosecha, gracias a la estacionalidad de ciertos productos.

Están condicionados a determinados cultivos y exclusivamente como riego complementario. Los permisos de uso de agua solo pueden utilizarse en cultivos de corto periodo vegetativo (lo que se denomina tallo corto), como pueden ser siembra de hortalizas y legumbres; más no en sembríos de tallo alto como árboles o frutales, pues no puede asegurarse que al año siguiente se cuente con el recurso. La referencia a que debe tratarse de un riego complementario, está relacionada a que un agricultor no puede confiar en que toda su producción, año tras año, sea realizada mediante un permiso de agua que puede nunca llegar a hacerse efectivo en caso de eventos climáticos extremos como una sequía.

También en esta oportunidad se recoge la precisión respecto a que los titulares de licencias de uso de agua, de cuyas aguas residuales se benefician los titulares de permisos, no son responsables de las consecuencias o de los perjuicios que puedan sobrevenir si variara la calidad, el caudal o volumen, u oportunidad, o si dejara de haber sobrantes de agua en cualquier momento o por cualquier motivo. Esta precisión era imprescindible que la recogiera la nueva ley, pues ofrece a los titulares de licencia de uso de agua que se constituyen en los aportantes de las aguas residuales, así como a la propia autoridad de aguas que otorga el derecho, un respaldo necesario ante cualquier cuestionamiento.

Durante años, a pesar de la claridad en las condiciones para ejercer el derecho de agua sustentado en permisos, los usuarios agrarios, particularmente, han ejercido presiones con tal de forzar la obtención de derechos de agua de carácter definitivo, como lo es una licencia. La estrategia más usada para forzar a las autoridades de agua al otorgamiento de licencias de uso de agua consistía en colocar cultivos permanentes y

37 Impresiona conocer que antes de la entrada en vigencia de la actual Ley de Recursos Hídricos existían valles de la costa norte del Perú eminentemente agrícolas, que en su totalidad dependían de la emisión anual de permisos de uso de agua, generando año a año una incertidumbre y preocupación respecto que les fuera o no otorgado el mismo.

EN TORNO AL

ACCESO AL AGUA

EN EL PERÚ, EN

EL MARCO DE

LA NUEVA LEY

DE RECURSOS

HÍDRICOS

THOUGHTS ON

ACCESS TO WATER

IN PERU WITHIN

THE NEW WATER

RESOURCES LAW

FRAMEWORK 
no temporales, como es el caso de árboles frutales por ejemplo. En caso no se les otorgara al siguiente año nuevamente un permiso, procedían a efectuar una denuncia contra la autoridad de aguas, en la que se argumentaba haber sufrido un perjuicio indemnizable. A pesar de que la Ley General de Aguas prohibía la instalación de cultivos permanentes sustentados en permisos de uso de agua y claramente establecía que no existía responsabilidad en caso los titulares de los permisos no pudieran ejercer sus derechos, algunos jueces por desconocimiento de la materia procedían a aceptar y en algunos casos incluso a sentenciar en favor del denunciante y en contra de la autoridad de aguas.

Con respecto al otorgamiento de los permisos de uso de agua, estos siempre han sido emitidos por las autoridades de agua de ámbito local, para lo cual con la actual legislación debe acreditarse ser propietario o poseedor legítimo del predio en el que se hará el uso eventual y contar en el predio de que se trate, con las obras autorizadas de captación, conducción, utilización, avenamiento, medición y las demás que fueran necesarias.

\section{AUTORIZACIONES DE USO DE AGUA}

\section{VI.1. Alcances de las autorizaciones de uso de agua}

La autorización de uso de agua originalmente estaba destinada a permitir la realización de estudios o ejecución de obras, así como a desarrollar otras labores de carácter transitorio y especial ${ }^{38}$. La Ley de Recursos Hídricos establece expresamente que este derecho otorga a su titular la facultad de usar una cantidad anual de agua para cubrir exclusivamente las necesidades derivadas o relacionadas directamente con la ejecución de estudios, la ejecución de obras o el lavado de suelos, restringiendo a estos tres supuestos su aplicación. Este derecho de uso se otorga por un plazo de dos años renovables por una única vez, por un periodo similar, siempre que subsistan las condiciones que dieron origen a su otorgamiento.

En el caso de las autorizaciones solicitadas para ejecutar estudios, el interesado debe mostrar que cuenta con un título de concesión otorgado por una entidad pública competente que tenga como fin la realización de estudios en cualquier actividad. Podemos afirmar, respecto de este supuesto, que más que usar el recurso, habrá una importante manipulación del mismo con la finalidad de determinar su volumen, características físico-químicas y periodicidad.

Quien requiera una autorización de agua para ejecutar obras debe contar con un certificado de aprobación de las mismas emitido por la autoridad 
competente. En este caso, debe considerarse que si los plazos otorgados mediante esta modalidad pueden ser como máximo de cuatro años, este no es un tipo de derecho que permita el desarrollo de grandes obras de infraestructura que requieren un plazo mayor para ser concluidas. Adicionalmente, queda claro que esta autorización permite solo el uso del recurso en el proceso de construcción, mas no otorga derechos para llevar adelante propiamente la actividad para la cual la obra hidráulica se está realizando. Finalmente, en caso se requiera una autorización para lavado de suelos ${ }^{39}$, el solicitante debe contar con el título de propiedad y un informe técnico que sustente la actividad, suscrito por perito.

A pesar de que la obtención de la autorización de uso de agua no compromete excesivamente al recurso hídrico al que se refiere, ni en tiempo ni en volumen, desde 1969 hasta 1981, este derecho se otorgaba mediante resolución ministerial ${ }^{40}$. Esto implicaba que obtener una autorización de uso de agua para realizar estudios de agua o ejecutar obras hidráulicas estuviera restringido al ámbito de las instituciones públicas. A partir de 1981, la autoridad de aguas desconcentró esta facultad a nivel regional e incluso loca ${ }^{41} \mathrm{y}$ actualmente este derecho es otorgado por la autoridad administrativa del agua.

\section{VI.2. Empleo inadecuado del término autorización de uso de agua}

Considerando los casos para los que se otorga una autorización de uso de agua, es claro que el volumen requerido es limitado, además de tratarse del desarrollo de actividades de una temporalidad corta. Sin embargo, existen diversos ejemplos de normativas sectoriales que han utilizado la denominación autorización de uso de agua en un sentido genérico y confundido la misma con el derecho que se requiere para realizar una actividad permanente.

Uno de los ejemplos más claros de esta inadecuada utilización del término autorización de uso de agua en sentido amplio se encuentra en la normatividad en materia de concesiones eléctricas de la década de $1990^{42}$, que planteaba como requisito para la obtención de la concesión definitiva de generación de energía eléctrica, que el interesado contara con la autorización del uso de recursos naturales de propiedad del Estado. La intención de la norma era que el desarrollador de un proyecto, que en este caso sería hidroenergético, contara con el elemento fundamental para llevar a cabo su actividad: el agua. Sin embargo, tal como se ha

39 El lavado del suelo es una técnica mediante la cual se usa generalmente agua para depurar el suelo, retirando, de esta manera, contaminantes peligrosos, concentrándolos y reduciendo su volumen. 40 Artículo 29 de la Ley General de Aguas, decreto ley 17752.

41 Decreto legislativo 106, decreto legislativo 653, decreto supremo 078-2006-AG, decreto legislativo 997 y ley 29338.

42 Ley de Concesiones Eléctricas dada por decreto ley 25844 y su reglamento aprobado mediante decreto supremo 009-93-EM.

REFLEXIONES

EN TORNO AL

ACCESO AL AGUA

EN EL PERÚ, EN

EL MARCO DE

LA NUEVA LEY

DE RECURSOS

HÍDRICOS

THOUGHTS ON

ACCESS TO WATER

IN PERU WITHIN

THE NEW WATER

RESOURCES LAW

FRAMEWORK 
mencionado, las autorizaciones de uso de agua son temporales y las actividades para las que se otorgan también lo son.

A lo que se refería la autoridad de energía de la década de 1990 era a que el inversionista debía cumplir con el requisito de tener asegurado el recurso hídrico para realizar la actividad, para lo cual debió referirse a la obtención de una licencia de uso de agua. Sin embargo, como también hemos mencionado, el otorgamiento de derechos de uso de agua se efectuaba para su uso efectivo y no podía obtenerse un derecho para no hacer uso de él, por lo que, aun cuando la autoridad de energía lo hubiera expresado de manera correcta («el solicitante debe obtener una licencia de uso de agua como requisito para obtener una concesión eléctrica»), la autoridad de aguas de la época no hubiera podido otorgar una licencia de uso de agua. Las opciones que se plantearon estaban más en la línea de lo que se denominaría una licencia condicionada ${ }^{43}$, figura jurídica que no existe en nuestra legislación.

\section{VII. ¿SON LAS LICENCIAS, AUTORIZACIONES Y PERMISOS LOS ÚNICOS DERECHOS DE USO DE AGUA?}

Es interesante que nuestra Ley de Recursos Hídricos haga mención a las comunidades campesinas y nativas y a las servidumbres, en el marco del capítulo que denomina «otros derechos de uso de agua» ${ }^{44}$, cuando en el primer caso se trata del reconocimiento de los derechos preferentes de las comunidades, pero no de una modalidad diferente de acceso al recurso, mientras que en el segundo caso estamos ante un gravamen sobre un predio para permitir el paso del recurso hídrico por él. Sin embargo, es en otro contexto donde encontramos un derecho respecto del agua, que no se ha considerado en los tres tipos que hemos analizado, a pesar de que sí implica autoridad respecto del recurso: la reserva de agua, que se encuentra ligada al desarrollo de infraestructura.

\section{VII.1. Reserva de uso de agua: un derecho especial}

La figura jurídica de la reserva de agua data de la Ley General de Aguas, en cuyo artículo 7 se mencionaba que el Poder Ejecutivo podía reservar un volumen de agua para cualquier finalidad de interés público, lo cual permitió que el gobierno peruano (único beneficiario de las mismas) reservara aguas para una serie de proyectos, fundamentalmente de infraestructura hidráulica de riego con miras a ampliar la frontera agrícola en la costa peruana. Estas reservas de dos años renovables ${ }^{45}$ podían estar vigentes durante décadas, imposibilitando la entrega 
física de estos recursos a terceros, en tanto la obra para la que se había solicitado no concluyera o la reserva se desestimara.

La Ley de Recursos Hídricos acoge la figura de la reserva de recursos hídricos ${ }^{46}$, definiéndola como un derecho especial intransferible que se otorga para el desarrollo de proyectos, que permite reservar un determinado volumen de agua de libre disponibilidad de una fuente natural de agua superficial o subterránea, con la finalidad de garantizar la atención de las demandas de un proyecto (público o privado) declarado de interés regional o nacional. El uso al que se destine puede ser consuntivo o no consuntivo y el periodo por el que se otorga esta reserva es de dos años renovables por el periodo de la elaboración de estudios y ejecución del proyecto separadamente, prorrogables en tanto subsistan las causas que los motivan.

Al igual que la figura original, creada en 1969, la reserva no faculta el uso del agua, sin embargo, en esta oportunidad, la Ley de Recursos Hídricos no circunscribe su otorgamiento a proyectos desarrollados por el Estado, ya que, de declararse de interés regional o nacional, puede otorgarse la reserva a un solicitante privado.

\section{LICENCIA PROVISIONAL, AUTORIZACIÓN DE USO Y RESERVA: ¿SE PUEDEN USAR INDISTINTAMENTE?}

Llama la atención apreciar las claras similitudes entre la licencia provisional, la autorización de usoy la reserva, basadas en su temporalidad, su búsqueda por interactuar con la inversión y apoyar el desarrollo de proyectos fundamentalmente de infraestructura. La percepción de que cualquiera de estas figuras se podría usar indistintamente para casos similares no es del todo cierta, por lo que debe darse una segunda mirada a sus alcances, esta vez de manera comparada, con el fin de apreciar similitudes y diferencias con las que comprenderemos cuándo usar o no cada modalidad.

\section{VIII.1. Plazos}

En los tres casos nos encontramos con figuras legales de plazo determinado, siendo dos años renovables el plazo planteado tanto en la autorización de uso como en la reserva de recurso hídrico, pero mayor es el que se refiere a la licencia provisional, en tanto este último caso está relacionado (y condicionado) al plazo de una concesión otorgada por algún sector productivo. En todos los casos, las renovaciones están sujetas a que se mantengan las condiciones existentes al momento de otorgar el derecho en primer lugar, pero, en el caso de las autorizaciones,

REFLEXIONES EN TORNO AL ACCESO AL AGUA EN EL PERÚ, EN EL MARCO DE LA NUEVA LEY DE RECURSOS HÍDRICOS

THOUGHTS ON ACCESS TO WATER IN PERU WITHIN THE NEW WATER RESOURCES LAW FRAMEWORK 
expresamente se habla de una única renovación, a diferencia de la reserva que no da plazo límite a las mismas.

\section{VIII.2. Uso efectivo del recurso}

Solo en el caso de las autorizaciones puede hacerse un uso efectivo del recurso establecido de manera anual, el cual no implica volúmenes significativos y en el caso de ejecución de estudios está más relacionado a la manipulación del recurso con miras a identificar características físicoquímicas, volumen y oportunidad de presencia del mismo que a un uso concreto y real. En el caso de las reservas y las licencias provisionales, sin embargo, no hay un uso efectivo del recurso, lo cual está expresamente establecido en el primer caso y tácitamente en el segundo ${ }^{47}$.

\section{VIII.3. Motivos para su otorgamiento}

En los casos de la licencia provisional y la autorización de uso, está involucrada la realización de estudios, tratándose en el primer caso de estudios en cualquier actividad autorizados a un concesionario, mientras que en el segundo se trata de estudios relacionados con el recurso agua propiamente. Por su parte, el establecimiento de una reserva de recurso hídrico está directamente relacionado con el desarrollo de infraestructura, pero al no poder hacer uso efectivo del recurso, si se quiere proceder a la ejecución de la obra, se requerirá la obtención de una autorización de uso de agua para ello.

\section{REFLEXIONES FINALES}

$\mathrm{Al}$ efectuar un primer acercamiento al tema hídrico, conocer las modalidades administrativas de acceso al recurso y la solidez de los derechos otorgados es un factor clave para entender las oportunidades y limitaciones que las diversas actividades humanas tienen respecto al agua.

De la presentación y análisis de los derechos de uso de agua, se puede apreciar que la Ley de Recursos Hídricos de 2008 acarreó cambios significativos en materia de modalidades de acceso al recurso hídrico, pero el mantener las mismas denominaciones que se usan desde hace cincuenta años, se puede generar una desinformación o confusión entre los mayores usuarios del recurso a nivel nacional que se asientan fundamentalmente en el ámbito rural. Esta situación nos muestra que un rediseño conceptual en el plano normativo requiere complementariamente la adecuada difusión a todo nivel respecto de los cambios realizados, con miras a prevenir conflictos futuros. 
Sea en el caso de las modalidades administrativas de acceso al agua, como en el de otros temas relacionados con la gestión integrada del recurso hídrico, en el Perú los análisis jurídicos sobre normativa de aguas deben hacerse de manera integral, visualizando tanto la Ley General de Aguas como la actual Ley de Recursos Hídricos. Es de esta manera que podrá afrontarse, al momento de la implementación de la norma, un diálogo en que los diversos actores tengan una comunicación clara y homogénea, logrando de esta manera consensos y no generando futuros conflictos.

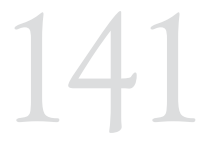

REFLEXIONES

EN TORNO AL

ACCESO AL AGUA

EN EL PERÚ, EN

EL MARCO DE

LA NUEVA LEY

DE RECURSOS

HIIDRICOS

Recibido: 09/01/2013

Aprobado: 11/03/2013
THOUGHTS ON

ACCESS TO WATER

IN PERU WITHIN

THE NEW WATER

RESOURCES LAW

FRAMEWORK 